\title{
Water loss from the skin of term and preterm babies
}

\author{
N. RUTTER AND D. HULL \\ Department of Child Health, University of Nottingham
}

SUMMARY Water loss from the skin of term and preterm babies, nursed naked in incubators under neutral thermal conditions, was measured by a method based on estimating the water vapour pressure gradient close to the skin surface. 199 sets of measurements were made on 78 babies whose gestational ages ranged from 26 to 41 weeks, during the first 4 weeks of life. Babies of 34 to 41 weeks' gestation had high water losses in the first 4 hours after birth, which then fell to low levels averaging $6 \mathrm{~g} / \mathrm{m}^{2}$ per hour. Babies of 30 to 33 weeks' gestation had high water losses in the first week which then fell to levels similar to those of mature babies. Babies $<30$ weeks' gestation had strikingly high losses, averaging $32 \mathrm{~g} / \mathrm{m}^{2}$ per hour in the first 4 days of life. At 2 weeks, levels were still higher than those of mature babies. Light-for-dates babies had skin water losses appropriate for their gestations. The high water losses in extremely preterm babies are probably transepidermal and the result of a thin, poorly keratinised stratum corneum. Water loss from the palms and soles was high in term babies and although low in preterm babies it rose steadily in the first 4 weeks of life. This is thought to represent the onset of emotional sweating. In terms of actual heat and water lost, skin water loss is relatively unimportant in term babies nursed naked under neutral thermal conditions. However, in babies $<30$ weeks' gestation, weighing $<1 \mathrm{~kg}$, skin water loss makes a major contribution to overall water balance. Furthermore, evaporative heat loss from the skin may exceed resting heat production. It is suggested that reduction of skin water loss in these babies may increase their chances of survival and their rates of growth.

Water loss from the skin of a newborn baby, either directly through the epidermis or from the sweat glands, may be important, firstly, as a source of heat loss and, secondly, as a major factor in overall water balance. Measurement of skin water loss from babies has been hampered in the past by the lack of a suitable method. Total insensible water loss from the skin and respiratory tract has been measured using a metabolic chamber (Hey and Katz, 1969; Zweymuller and Preining, 1970), or by measurement of weight loss (Levine et al., 1930; Fanaroff et al., 1972; Wu and Hodgman, 1974; Marks et al., 1977). Measurements of skin water loss from isolated sites have been made using a ventilated capsule (Foster et al., 1969; Cunico et al., 1977). Results suggest that skin water loss is low in term babies, but that in preterm ones, whether nursed naked in standard incubators or under radiant warmers or phototherapy, losses are high.

Department of Child Health, Medical School, Queen's Medical Centre, Nottingham

N. RUTTER, senior lecturer in child health

D. HULL, professor of child health
Nilsson (1977) described a method for measuring water loss from the skin, based on estimation of water vapour pressure gradient close to the skin surface. Hammarlund et al. (1977) used this method to measure transepidermal water loss in term infants nursed naked in incubators under neutral thermal conditions and found that it decreased as the ambient humidity was increased. They also measured rates of evaporation from several sites in a small number of babies on the first day of life and found that while rates were high on the face and extremities, they were generally low elsewhere. We were interested in using this simple method to examine the rates of skin water loss from different parts of the body in both term and preterm infants, and to see how they changed with postnatal age.

\section{Method}

Evaporation rate (ER) of water from the skin was measured using the commerically available apparatus devised by Nilsson, the Evaporimeter EP1 (Servomed). This measures the water vapour 
pressure gradient close to the skin surface which is directly proportional to ER. The method has been described in detail (Nilsson, 1977). The relative humidity sensors were calibrated by placing the probe in atmospheres of known humidity.

Measurements were made on naked babies in a standard single-walled incubator with a proportionally controlled heater ( 59 or 79 , Vickers Medical) the air temperature of which was set close to the lower end of the thermoneutral range, according to the values reported by Hey (1971). Several very low birthweight babies in the first week of life required incubator air temperatures in excess of $36.9^{\circ} \mathrm{C}$ to maintain normal rectal temperatures, these temperatures being the maximum obtainable when using the incubator in the air mode. In these cases, servo-control was used, setting the abdominal skin temperatures to $36 \cdot 5-36 \cdot 9^{\circ} \mathrm{C}$ and allowing the air temperature to rise to $39^{\circ} \mathrm{C}$ if necessary. Measurements were made on the quiet or sleeping baby through the portholes of the incubator at least 20 minutes after any adjustment to incubator temperature. The ER from a small area of skin (approximately $1 \mathrm{~cm}^{2}$ ) was measured by lightly resting the probe on the baby's skin until a stable reading was obtained. To avoid errors due to moisture on the investigator's hands, rubber gloves were worn. ER in $\mathrm{g} / \mathrm{m}^{2}$ per hour was measured at 18 different skin sites in all babies: forehead and cheek representing the head $(21 \%)$, chest and abdomen $(14 \%)$, back $(13 \%)$, buttock $(5 \%)$, anterior and posterior surfaces of the upper arm (8\%), and lower arm $(6 \%)$, palm and dorsum of the hand $(5 \%)$, anterior and posterior surfaces of the thigh $(11 \%)$, and lower leg $(10 \%)$, and the sole and dorsum of the foot $(7 \%)$. The figures in brackets represent the percentage of the total surface area contributed by the various sites in newborn babies (Lund and Browder, 1944).

The rectal temperature was measured in term babies with a mercury thermometer inserted approximately $4 \mathrm{~cm}$ into the rectum and in preterm babies with a thermocouple probe inserted approximately $8 \mathrm{~cm}$ into the baby's rectum. The relative humidity within the incubator was not controlled but was measured with the Evaporimeter probe and recorded. The incubator air temperature was measured with a thermocouple placed in the centre of the incubator. Skin temperature was measured by a thermocouple lightly taped to the upper abdomen. The gestational age of the baby was calculated in completed weeks of pregnancy from the first day of the mother's last menstrual period. In addition, in the preterm babies (37 weeks' gestation or less) a gestational assessment was carried out using a scoring system based on external characteristics (Farr et al.,
1966). Results were not included if there was a wide discrepancy between the assessed gestational age and that based on the mother's menstrual data. Babies were judged either appropriate or light-for-dates using the 10th centile on the charts of Babson et al. (1970) and Thomson et al. (1968).

\section{Subjects}

The babies were divided into four groups according to gestational age.

Group 1 (38-41 weeks). 57 sets of measurements were made on 36 healthy, term babies, all appropriate for dates, aged from 2 hours to 10 days. Mean birthweight was $3 \cdot 31 \mathrm{~kg}$ (range $2 \cdot 60-4 \cdot 36$ ).

Group 2 (34-37 weeks). 29 sets of measurements were made on 16 healthy babies, aged from 2 hours to 21 days. Nine babies were light-for-dates. Mean birthweight was $2 \cdot 08 \mathrm{~kg}$ (range $1 \cdot 07-2 \cdot 90$ ).

Group 3 (30-33 weeks). 65 sets of measurements were made on 16 healthy babies, aged from 2 hou:s to 27 days. Four babies were light-for-dates. Mean birthweight was $1 \cdot 43 \mathrm{~kg}$ (range $1 \cdot 01-2 \cdot 20$ ).

Group 4 ( $<30$ weeks). 48 sets of measurements were made on 10 babies, aged from one huur to 25 days. All were appropriate for dates. Eight of the 10 babies were unwell with either recurrent apnoea or respiratory distress syndrome during at least one of the sets of measurements. They were receiving oxygen and intravenous fluids. Six babies were receiving intermittent positive pressure ventilation when studied. Six of the 10 babies died. Mean birthweight was $0.89 \mathrm{~kg}$ (range $0 \cdot 66-1 \cdot 34$ ). Gestational ages ranged from 26 to 29 weeks.

\section{Results}

The babies were studied at ambient temperatures judged to be close to the lower end of the thermoneutral range (Table 1). Room air temperature varied between 25 and $30^{\circ} \mathrm{C}$ and thus the operative environmental temperatures, allowing for radiant heat exchanges, were probably about $1^{\circ} \mathrm{C}$ less than the actual incubator air temperatures (Hey and Mount, 1966). The mean abdominal skin and rectal temperatures of babies below 30 weeks' gestation in the first week of life were lower than those of more mature infants in spite of high incubator air temperatures and, in many instances, the baby's surface and rectal temperatures were below that of the surrounding air. Relative humidity varied between 27 and $63 \%$ and mean values in each group of babies were similar. 
Table 1 Mean incubator air, abdominal skin and rectal temperatures, and mean ambient relative humidity in the four groups of babies

\begin{tabular}{|c|c|c|c|c|c|c|c|c|c|c|c|c|c|}
\hline \multirow[t]{2}{*}{ Group } & \multirow{2}{*}{$\begin{array}{l}\text { Postnatal } \\
\text { age } \\
\text { (days) }\end{array}$} & \multicolumn{3}{|c|}{$\begin{array}{l}\text { Incubator air temperature } \\
\left({ }^{\circ} \mathrm{C}\right)\end{array}$} & \multicolumn{3}{|c|}{$\begin{array}{l}\text { Abdominal skin } \\
\text { temperature }\left({ }^{\circ} \mathrm{C}\right)\end{array}$} & \multicolumn{3}{|c|}{$\begin{array}{l}\text { Rectal temperature } \\
\left({ }^{\circ} \mathrm{C}\right)\end{array}$} & \multicolumn{3}{|c|}{$\begin{array}{l}\text { Relative humidity of } \\
\text { incubator air } \%\end{array}$} \\
\hline & & Mean & $S D$ & Range & Mean & $S D$ & Range & Mean & $S D$ & Range & Mean & $S D$ & Range \\
\hline $\begin{array}{c}1 \text { (38-41 } \\
\text { weeks) } \\
2 \text { (34-37 } \\
\text { weeks) } \\
3 \text { (30-33 } \\
\text { weeks) } \\
4 \text { (<30 } \\
\text { weeks) }\end{array}$ & $\begin{array}{l}0-11 \\
0-6 \\
7-21 \\
0-6 \\
7-27 \\
0-6 \\
7-13 \\
14-25\end{array}$ & $\begin{array}{l}33 \cdot 1 \\
34 \cdot 5 \\
33 \cdot 5 \\
35 \cdot 7 \\
34 \cdot 0 \\
37 \cdot 4 \\
36 \cdot 7 \\
34 \cdot 8\end{array}$ & $\begin{array}{l}0 \cdot 5 \\
1 \cdot 2 \\
0 \cdot 7 \\
1 \cdot 0 \\
1 \cdot 4 \\
1 \cdot 5 \\
2 \cdot 4 \\
2 \cdot 5\end{array}$ & $\begin{array}{l}32 \cdot 0-34 \cdot 5 \\
33 \cdot 1-36 \cdot 6 \\
32 \cdot 5-34 \cdot 5 \\
32 \cdot 7-37 \cdot 1 \\
31 \cdot 6-36 \cdot 7 \\
34 \cdot 0-40 \cdot 1 \\
31 \cdot 1-39 \cdot 5 \\
31 \cdot 2-38 \cdot 5\end{array}$ & $\begin{array}{l}35 \cdot 8 \\
36 \cdot 4 \\
36 \cdot 8 \\
36 \cdot 8 \\
36 \cdot 7 \\
36 \cdot 3 \\
36 \cdot 7 \\
36 \cdot 7\end{array}$ & $\begin{array}{l}0.5 \\
0.5 \\
0.5 \\
0.4 \\
0.4 \\
1.0 \\
0.5 \\
0.7\end{array}$ & $\begin{array}{l}34 \cdot 8-36 \cdot 8 \\
35 \cdot 2-37 \cdot 0 \\
36 \cdot 0-37 \cdot 5 \\
35 \cdot 9-37 \cdot 9 \\
35 \cdot 7-37 \cdot 7 \\
33 \cdot 3-37 \cdot 6 \\
35 \cdot 9-37 \cdot 4 \\
36 \cdot 0-37 \cdot 7\end{array}$ & $\begin{array}{l}37 \cdot 0 \\
36 \cdot 9 \\
37 \cdot 3 \\
37 \cdot 1 \\
37 \cdot 2 \\
36 \cdot 7 \\
37 \cdot 1 \\
37 \cdot 1\end{array}$ & $\begin{array}{l}0.4 \\
0.5 \\
0.4 \\
0.4 \\
0.3 \\
0.9 \\
0.4 \\
0.6\end{array}$ & $\begin{array}{l}35 \cdot 8-37 \cdot 8 \\
35 \cdot 7-37 \cdot 4 \\
36 \cdot 7-37 \cdot 7 \\
36 \cdot 3-38 \cdot 3 \\
36 \cdot 7-37 \cdot 8 \\
33 \cdot 7-38 \cdot 4 \\
36-6-37 \cdot 9 \\
35 \cdot 9-37 \cdot 9\end{array}$ & $\begin{array}{l}47 \\
42 \\
41 \\
44 \\
43 \\
42 \\
39 \\
46\end{array}$ & $\begin{array}{r}5 \\
4 \\
6 \\
7 \\
5 \\
5 \\
7 \\
12\end{array}$ & $\begin{array}{l}33-60 \\
37-55 \\
29-48 \\
30-59 \\
28-55 \\
33-56 \\
28-47 \\
27-63\end{array}$ \\
\hline
\end{tabular}

Overall skin water loss. An overall water loss, representative of the loss from the baby's skin as a whole, was obtained by multiplying each of the 18 measurements of ER by the percentage contribution that the measurement site made to total surface area and then adding these together. Results were examined for each group of babies.

Group 1 (Fig. 1)

Water losses in this group were low. Measurements made in the first 4 hours averaged $10.5 \mathrm{~g} / \mathrm{m}^{2}$ per hour

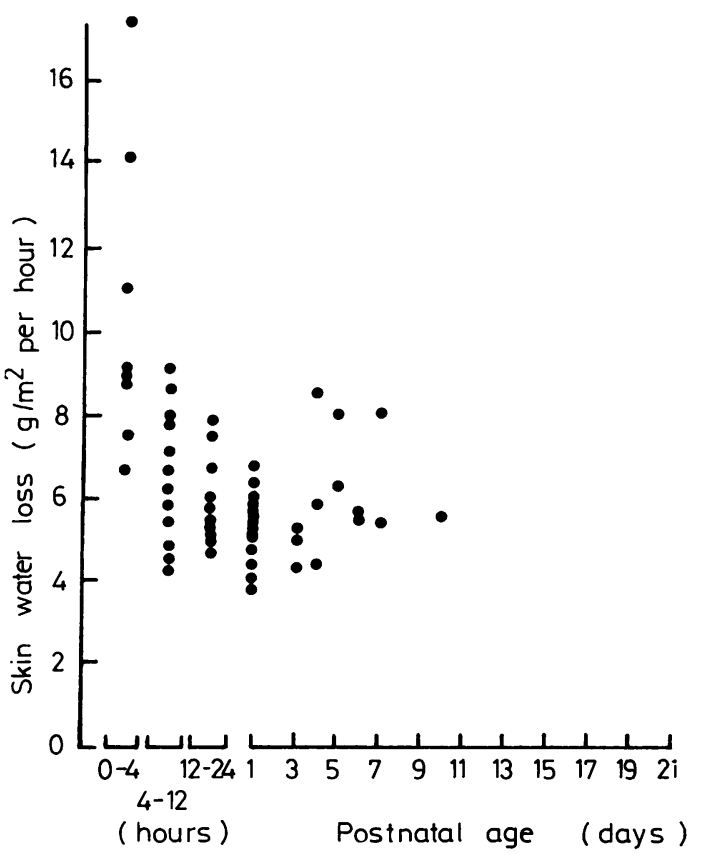

Fig. 1 Skin water loss for babies of 38 to 41 weeks' gestation. but after this values fell. Average skin water loss after the first 4 hours was $5 \cdot 9 \pm 1 \cdot 3 \mathrm{~g} / \mathrm{m}^{2}$ per hour (mean $\pm \mathrm{SD}$ ).

Group 2 (Fig. 2)

Water losses in this group were also low and values were similar to those found in term babies. Measurements made in the first 4 hours averaged 11.0 $\mathrm{g} / \mathrm{m}^{2}$ per hour. After this time the average skin water loss was $6 \cdot 0 \pm 1 \cdot 2 \mathrm{~g} / \mathrm{m}^{2}$ per hour. Babies who were light-for-dates did not differ from those who were appropriate for dates.

\section{Group 3 (Fig. 3)}

Skin water losses were considerably higher than in term babies and the fall in values after 4 hours of age was not seen. Average losses in the first week were $13.0 \pm 3.5 \mathrm{~g} / \mathrm{m}^{2}$ per hour, but from the second week onwards were similar to those in term babies $\left(7.8 \pm 1.7 \mathrm{~g} / \mathrm{m}^{2}\right.$ per hour). Babies who were light-for-dates did not differ from those who were appropriate for dates.

\section{Group 4 (Fig. 4)}

Skin water losses in these extremely preterm babies were very much higher than those seen in term ones, and again the fall in values after 4 hours of age was not seen. In the first 3 days losses averaged $32 \cdot 4 \pm 13 \cdot 6 \mathrm{~g} / \mathrm{m}^{2}$ per hour. Losses were still high after the first week; 3 out of 5 babies studied serially at this time had losses 2 to 4 times greater than those of mature babies. Average losses from 4 to 7 days of age were $21 \cdot 5 \pm 8 \cdot 0 \mathrm{~g} / \mathrm{m}^{2}$ per hour, from 8 to 14 days $15 \cdot 7 \pm 7 \cdot 6 \mathrm{~g} / \mathrm{m}^{2}$ per hour, and from 15 days onwards $11 \cdot 4 \pm 4 \cdot 8 \mathrm{~g} / \mathrm{m}^{2}$ per hour.

Water loss from individual sites (Table 2). As would be expected, the rates of evaporation at individual sites fell as both gestational age and postnatal age 


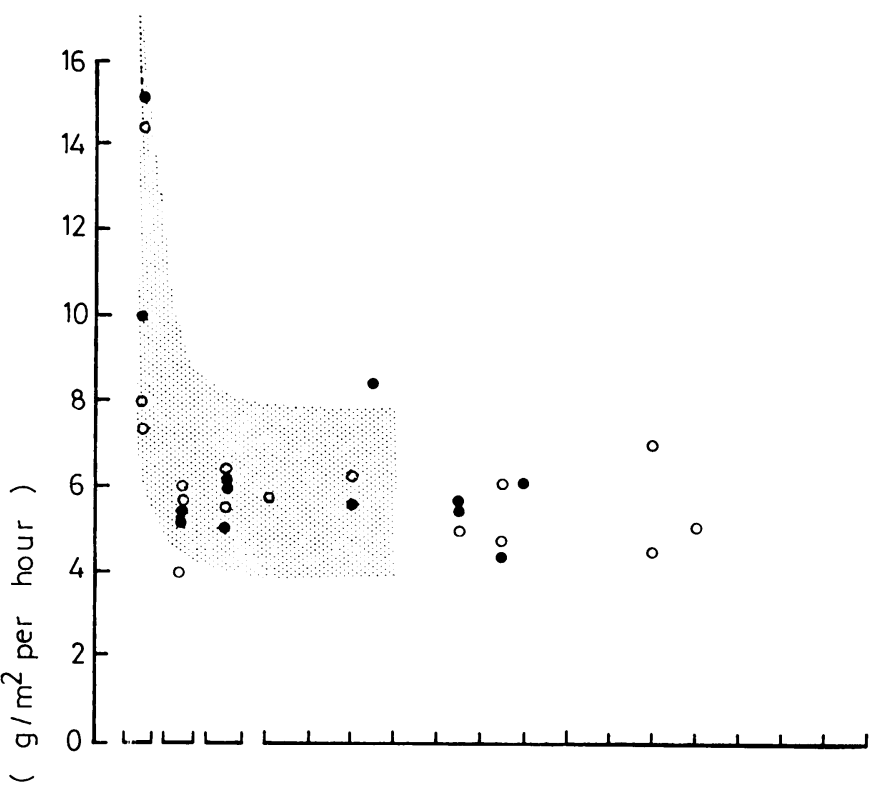

Fig. 2 Skin water loss for babies of 34 to 37 weeks' gestation. Upper figure shows all the measurements made on babies in this group. Lower figure shows those measurements which were made serially on the same baby, Hatched area represents the range of skin water loss found in term babies (Fig. 1).

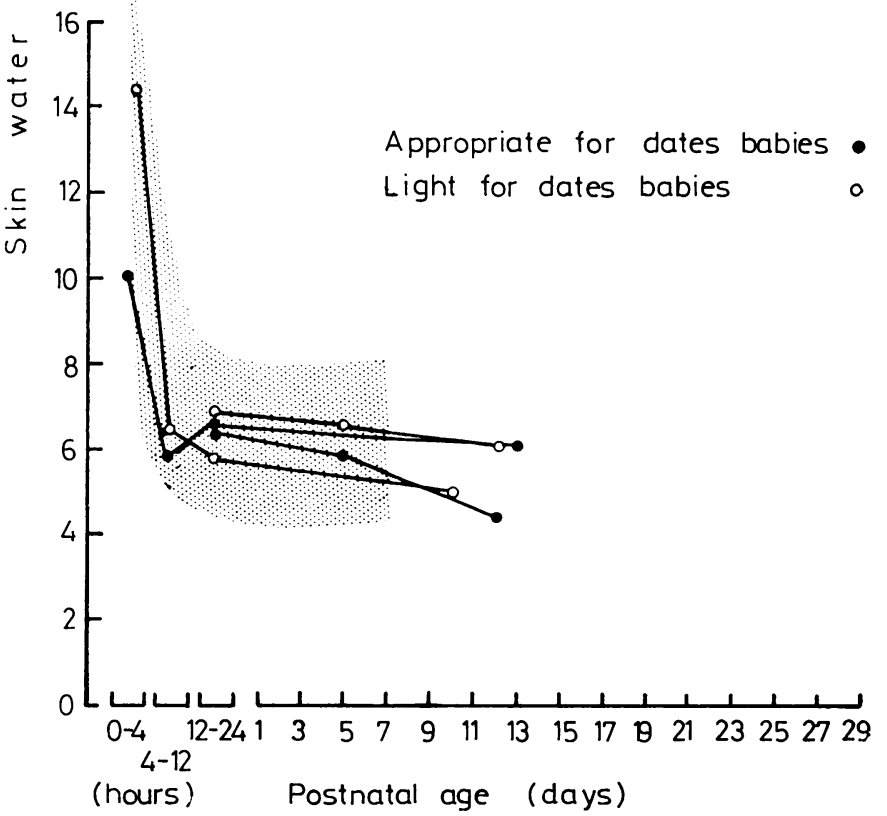




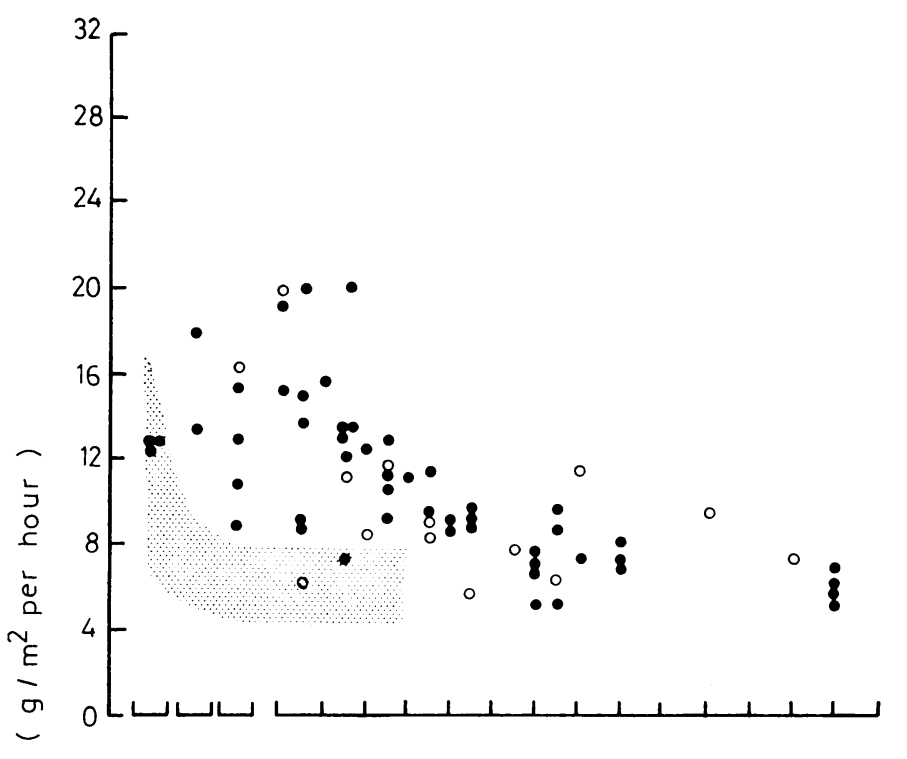



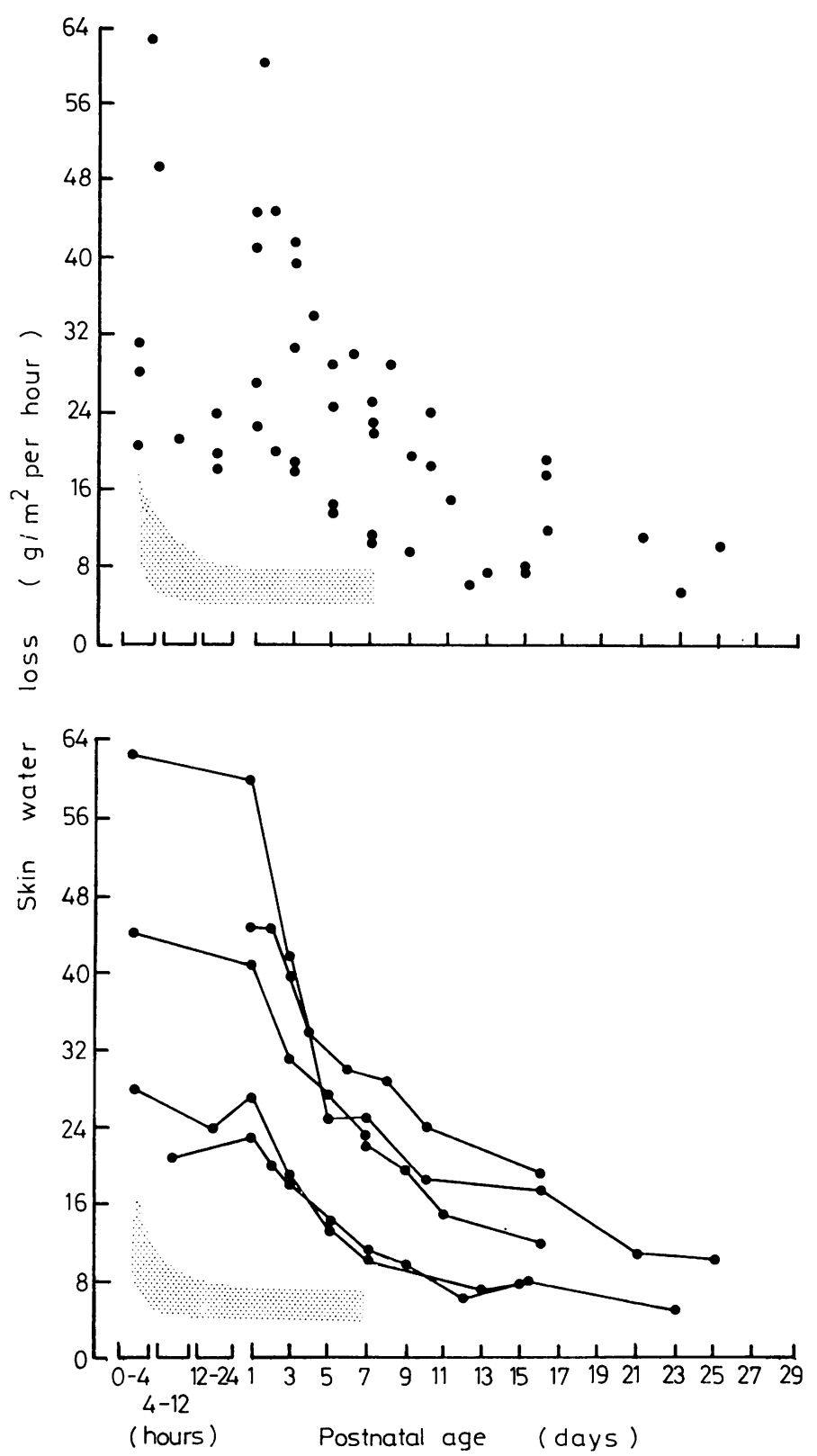

Fig. 4 Skin water loss for babies $<30$ weeks' gestation.

increased. A number of interesting findings emerged. In the most immature group of babies (group 4) the highest rate of water loss was found on the abdomen (Fig. 5). Lowest losses were found on the cheek, forehead, palm, and sole. As the babies grew older, all losses fell with the exception of those from the palm and the sole where water loss actually increased. A similar rise in water loss from these sites as the babies grew older was seen in the other preterm babies (groups 2 and 3). Term babies (group 1) had low rates of evaporation over most of the body but water losses from the palms and the soles were high. 
864 Rutter and Hull

Table 2 Mean rates of evaporation at 18 different skin sites according to the gestational and postnatal age of the baby $\left(\mathrm{g} / \mathrm{m}^{2}\right.$ per hour $)$

\begin{tabular}{|c|c|c|c|c|c|c|c|c|c|c|c|c|}
\hline \multirow{2}{*}{$\begin{array}{l}\text { Gestational age } \\
\text { (weeks) }\end{array}$} & \multicolumn{3}{|c|}{ 0-6 days } & \multicolumn{3}{|c|}{ 7-13 days } & \multicolumn{3}{|c|}{ 14-20 days } & \multicolumn{3}{|c|}{$21-27$ days } \\
\hline & Mean & $S D$ & $n$ & Mean & $S D$ & $n$ & Mean & $S D$ & $n$ & Mean & $S D$ & $n$ \\
\hline $\begin{array}{c}\text { Forehead } \\
\quad<30 \\
30-33 \\
34-37 \\
38-41\end{array}$ & $\begin{array}{r}14 \cdot 4 \\
9 \cdot 0 \\
12 \cdot 2 \\
6 \cdot 7\end{array}$ & $\begin{array}{r}6 \cdot 2 \\
3 \cdot 7 \\
16 \cdot 5 \\
3 \cdot 8\end{array}$ & $\begin{array}{l}27 \\
34 \\
19 \\
54\end{array}$ & $\begin{array}{r}10 \cdot 0 \\
6 \cdot 3 \\
5 \cdot 1 \\
-\end{array}$ & $\begin{array}{l}5 \cdot 7 \\
2 \cdot 0 \\
1.6 \\
-\end{array}$ & $\begin{array}{r}12 \\
17 \\
8 \\
-\end{array}$ & $\begin{array}{l}7.4 \\
8.0 \\
- \\
-\end{array}$ & $\begin{array}{l}2.5 \\
3.7 \\
-\end{array}$ & $\begin{array}{l}5 \\
8 \\
- \\
-\end{array}$ & $\begin{array}{l}4.7 \\
5 \cdot 3 \\
-\end{array}$ & $\begin{array}{l}\overline{1 \cdot 4} \\
-\end{array}$ & $\begin{array}{l}3 \\
6 \\
- \\
\end{array}$ \\
\hline $\begin{array}{l}\text { Cheek } \\
<30 \\
30-33 \\
34-37 \\
38-41\end{array}$ & $\begin{array}{r}19 \cdot 3 \\
9.7 \\
5 \cdot 7 \\
5 \cdot 9\end{array}$ & $\begin{array}{l}8 \cdot 9 \\
3 \cdot 3 \\
2 \cdot 3 \\
1 \cdot 8\end{array}$ & $\begin{array}{l}27 \\
34 \\
19 \\
54\end{array}$ & $\begin{array}{r}12 \cdot 3 \\
6 \cdot 4 \\
5 \cdot 9 \\
-\end{array}$ & $\begin{array}{l}6 \cdot 8 \\
1 \cdot 5 \\
3 \cdot 1 \\
-\end{array}$ & $\begin{array}{r}13 \\
17 \\
8 \\
-\end{array}$ & $\begin{array}{c}10.8 \\
6.8 \\
-\end{array}$ & $\begin{array}{l}\overline{2 \cdot 4} \\
- \\
-\end{array}$ & $\begin{array}{l}4 \\
9 \\
- \\
-\end{array}$ & $\begin{array}{l}4 \cdot 3 \\
5 \cdot 5 \\
-\end{array}$ & $\begin{array}{l}\overline{1 \cdot 1} \\
- \\
-\end{array}$ & $\begin{array}{l}3 \\
6 \\
- \\
\end{array}$ \\
\hline $\begin{array}{l}\text { Chest } \\
\qquad 30 \\
\begin{array}{c}30-33 \\
34-37 \\
38-41\end{array}\end{array}$ & $\begin{array}{r}25 \cdot 9 \\
11 \cdot 2 \\
6 \cdot 1 \\
6 \cdot 0\end{array}$ & $\begin{array}{r}12 \cdot 8 \\
3 \cdot 5 \\
2 \cdot 0 \\
2 \cdot 3\end{array}$ & $\begin{array}{l}26 \\
32 \\
19 \\
54\end{array}$ & $\begin{array}{r}17 \cdot 0 \\
7 \cdot 5 \\
5 \cdot 1 \\
-\end{array}$ & $\begin{array}{l}7 \cdot 0 \\
2 \cdot 3 \\
1.4 \\
-\end{array}$ & $\begin{array}{r}14 \\
17 \\
8 \\
-\end{array}$ & $\begin{array}{c}14 \cdot 6 \\
7 \cdot 2 \\
- \\
-\end{array}$ & $\begin{array}{l}7 \cdot 1 \\
2 \cdot 1 \\
- \\
-\end{array}$ & $\begin{array}{l}5 \\
9 \\
- \\
-\end{array}$ & $\begin{array}{l}8 \cdot 7 \\
6 \cdot 3 \\
-\end{array}$ & $\begin{array}{l}\overline{0.5} \\
- \\
-\end{array}$ & $\begin{array}{l}3 \\
6 \\
-\end{array}$ \\
\hline $\begin{array}{c}\text { Abdomen } \\
<30 \\
30-33 \\
34-37 \\
38-41\end{array}$ & $\begin{array}{r}51 \cdot 1 \\
15 \cdot 2 \\
7 \cdot 7 \\
5 \cdot 7\end{array}$ & $\begin{array}{r}30.5 \\
5.8 \\
1.8 \\
1.9\end{array}$ & $\begin{array}{l}27 \\
34 \\
19 \\
54\end{array}$ & $\begin{array}{r}23 \cdot 0 \\
8 \cdot 9 \\
5 \cdot 9 \\
-\end{array}$ & $\begin{array}{r}12 \cdot 7 \\
2 \cdot 3 \\
1 \cdot 9 \\
-\end{array}$ & $\begin{array}{r}14 \\
16 \\
8 \\
-\end{array}$ & $\begin{array}{c}16.4 \\
9.9 \\
- \\
-\end{array}$ & $\begin{array}{l}4 \cdot 7 \\
3 \cdot 2 \\
- \\
-\end{array}$ & $\begin{array}{l}5 \\
8 \\
- \\
-\end{array}$ & $\begin{array}{l}10.0 \\
7.8 \\
-\end{array}$ & $\begin{array}{l}\overline{1 \cdot 9} \\
- \\
-\end{array}$ & $\begin{array}{l}3 \\
6 \\
-\end{array}$ \\
\hline $\begin{array}{l}\text { Back } \\
\qquad 30 \\
\begin{array}{c}30-33 \\
34-37 \\
38-41\end{array}\end{array}$ & $\begin{array}{r}40 \cdot 5 \\
14 \cdot 2 \\
7 \cdot 3 \\
5 \cdot 8\end{array}$ & $\begin{array}{r}25 \cdot 3 \\
4 \cdot 8 \\
2 \cdot 3 \\
1 \cdot 9\end{array}$ & $\begin{array}{l}26 \\
34 \\
18 \\
54\end{array}$ & $\begin{array}{r}17.4 \\
7.1 \\
4.9 \\
-\end{array}$ & $\begin{array}{l}8 \cdot 7 \\
1.8 \\
1.8 \\
-\end{array}$ & $\begin{array}{r}14 \\
17 \\
8 \\
-\end{array}$ & $\begin{array}{c}12 \cdot 4 \\
6 \cdot 2 \\
-\end{array}$ & $\begin{array}{l}8 \cdot 3 \\
1 \cdot 4 \\
- \\
-\end{array}$ & $\begin{array}{l}5 \\
8 \\
- \\
-\end{array}$ & $\begin{array}{l}8.0 \\
5 \cdot 5 \\
-\end{array}$ & $\begin{array}{l}\overline{1 \cdot 1} \\
-\end{array}$ & $\begin{array}{l}3 \\
6 \\
- \\
\end{array}$ \\
\hline $\begin{array}{l}\text { Upper arm-front } \\
<30 \\
30-33 \\
34-37 \\
38-41\end{array}$ & $\begin{array}{r}36 \cdot 2 \\
15 \cdot 7 \\
7 \cdot 6 \\
8 \cdot 1\end{array}$ & $\begin{array}{r}17 \cdot 8 \\
5 \cdot 1 \\
3 \cdot 8 \\
6 \cdot 2\end{array}$ & $\begin{array}{l}27 \\
34 \\
19 \\
54\end{array}$ & $\begin{array}{r}19 \cdot 2 \\
9.5 \\
4.9 \\
-\end{array}$ & $\begin{array}{l}9 \cdot 2 \\
2 \cdot 9 \\
2 \cdot 7 \\
-\end{array}$ & $\begin{array}{r}14 \\
17 \\
8 \\
-\end{array}$ & $\begin{array}{c}16.6 \\
7.9 \\
-\end{array}$ & $\begin{array}{l}8 \cdot 3 \\
2 \cdot 8 \\
- \\
-\end{array}$ & $\begin{array}{l}5 \\
9 \\
- \\
-\end{array}$ & $\begin{array}{c}13 \cdot 7 \\
6 \cdot 2 \\
- \\
-\end{array}$ & $\begin{array}{l}\overline{1 \cdot 8} \\
- \\
-\end{array}$ & $\begin{array}{l}3 \\
6 \\
- \\
\end{array}$ \\
\hline $\begin{array}{l}\text { Lower arm-front } \\
<30 \\
30-33 \\
34-37 \\
38-41\end{array}$ & $\begin{array}{r}37 \cdot 7 \\
16 \cdot 3 \\
7 \cdot 4 \\
6 \cdot 4\end{array}$ & $\begin{array}{r}19.0 \\
5.0 \\
2.4 \\
3 \cdot 1\end{array}$ & $\begin{array}{l}27 \\
34 \\
19 \\
53\end{array}$ & $\begin{array}{r}22 \cdot 1 \\
10.8 \\
5.8 \\
-\end{array}$ & $\begin{array}{r}13 \cdot 3 \\
4 \cdot 5 \\
2 \cdot 4 \\
-\end{array}$ & $\begin{array}{r}14 \\
17 \\
8 \\
-\end{array}$ & $\begin{array}{c}16.2 \\
9.8 \\
-\end{array}$ & $\begin{array}{l}6 \cdot 4 \\
3 \cdot 0 \\
- \\
-\end{array}$ & $\begin{array}{l}5 \\
9 \\
- \\
-\end{array}$ & $\begin{array}{c}16 \cdot 0 \\
6 \cdot 5 \\
- \\
-\end{array}$ & $\begin{array}{l}\overline{1 \cdot 4} \\
- \\
-\end{array}$ & $\begin{array}{l}3 \\
6 \\
- \\
\end{array}$ \\
\hline $\begin{array}{l}\text { Lower leg-front } \\
\quad<30 \\
30-33 \\
34-37 \\
38-41\end{array}$ & $\begin{array}{r}36 \cdot 1 \\
15 \cdot 1 \\
6 \cdot 5 \\
5 \cdot 2\end{array}$ & $\begin{array}{r}16 \cdot 1 \\
5 \cdot 1 \\
1 \cdot 9 \\
1 \cdot 8\end{array}$ & $\begin{array}{l}26 \\
34 \\
19 \\
54\end{array}$ & $\begin{array}{r}17 \cdot 6 \\
9 \cdot 1 \\
5 \cdot 7 \\
-\end{array}$ & $\begin{array}{l}9.6 \\
3.0 \\
1.8 \\
-\end{array}$ & $\begin{array}{r}14 \\
17 \\
8 \\
-\end{array}$ & $\begin{array}{c}11 \cdot 0 \\
7 \cdot 1 \\
- \\
-\end{array}$ & $\begin{array}{l}3 \cdot 2 \\
2 \cdot 5 \\
- \\
-\end{array}$ & $\begin{array}{l}5 \\
8 \\
- \\
-\end{array}$ & $\begin{array}{c}10.7 \\
5.5 \\
- \\
-\end{array}$ & $\begin{array}{l}\overline{1 \cdot 1} \\
-\end{array}$ & $\begin{array}{l}3 \\
6 \\
- \\
\end{array}$ \\
\hline $\begin{array}{l}\text { Lower leg-back } \\
\quad<30 \\
\begin{array}{l}30-33 \\
34-37 \\
38-41\end{array}\end{array}$ & $\begin{array}{r}35.4 \\
16 \cdot 1 \\
5 \cdot 9 \\
4 \cdot 6\end{array}$ & $\begin{array}{r}16 \cdot 2 \\
6 \cdot 9 \\
1 \cdot 4 \\
1 \cdot 3\end{array}$ & $\begin{array}{l}26 \\
33 \\
18 \\
54\end{array}$ & $\begin{array}{r}15 \cdot 2 \\
8 \cdot 5 \\
5 \cdot 5 \\
-\end{array}$ & $\begin{array}{l}7.4 \\
2.4 \\
1.8 \\
-\end{array}$ & $\begin{array}{r}14 \\
17 \\
8 \\
-\end{array}$ & $\begin{array}{c}14 \cdot 1 \\
7 \cdot 1 \\
- \\
-\end{array}$ & $\begin{array}{l}7 \cdot 1 \\
2 \cdot 1 \\
- \\
-\end{array}$ & $\begin{array}{l}5 \\
8 \\
- \\
-\end{array}$ & $\begin{array}{c}11.0 \\
5.5 \\
-\end{array}$ & $\begin{array}{l}\overline{1 \cdot 1} \\
- \\
-\end{array}$ & $\begin{array}{l}3 \\
6 \\
-\end{array}$ \\
\hline $\begin{array}{l}\text { Palm } \\
\qquad 30 \\
30-33 \\
34-37 \\
38-41\end{array}$ & $\begin{array}{l}16 \cdot 8 \\
11 \cdot 9 \\
13.0 \\
26 \cdot 5\end{array}$ & $\begin{array}{r}6 \cdot 6 \\
6 \cdot 4 \\
3 \cdot 8 \\
16 \cdot 3\end{array}$ & $\begin{array}{l}27 \\
34 \\
19 \\
53\end{array}$ & $\begin{array}{l}16 \cdot 6 \\
13 \cdot 3 \\
14 \cdot 3 \\
-\end{array}$ & $\begin{array}{l}6 \cdot 4 \\
4 \cdot 7 \\
5 \cdot 2 \\
-\end{array}$ & $\begin{array}{r}14 \\
16 \\
8 \\
-\end{array}$ & $\begin{array}{l}17 \cdot 4 \\
14 \cdot 8 \\
-\end{array}$ & $\begin{array}{l}6 \cdot 8 \\
6 \cdot 1 \\
- \\
-\end{array}$ & $\begin{array}{l}5 \\
9 \\
- \\
-\end{array}$ & $\begin{array}{l}21 \cdot 7 \\
13 \cdot 7 \\
- \\
-\end{array}$ & $\begin{array}{l}\overline{3 \cdot 1} \\
- \\
-\end{array}$ & $\begin{array}{l}3 \\
6 \\
-\end{array}$ \\
\hline $\begin{array}{l}\text { Upper arm-back } \\
<30 \\
30-33 \\
34-37 \\
38-41\end{array}$ & $\begin{array}{r}31 \cdot 2 \\
11 \cdot 9 \\
5 \cdot 7 \\
5 \cdot 1\end{array}$ & $\begin{array}{r}15 \cdot 3 \\
4 \cdot 6 \\
1 \cdot 8 \\
2 \cdot 2\end{array}$ & $\begin{array}{l}26 \\
34 \\
18 \\
54\end{array}$ & $\begin{array}{r}14.2 \\
7.9 \\
4.7 \\
-\end{array}$ & $\begin{array}{l}6 \cdot 4 \\
2.0 \\
1 \cdot 7 \\
-\end{array}$ & $\begin{array}{r}14 \\
17 \\
8 \\
-\end{array}$ & $\begin{array}{c}11.6 \\
7.0 \\
-\end{array}$ & $\begin{array}{l}5 \cdot 0 \\
3 \cdot 0 \\
- \\
-\end{array}$ & $\begin{array}{l}5 \\
9 \\
- \\
-\end{array}$ & $\begin{array}{l}7 \cdot 3 \\
4 \cdot 7 \\
- \\
-\end{array}$ & $\begin{array}{l}\overline{0.8} \\
- \\
-\end{array}$ & $\begin{array}{l}3 \\
6 \\
-\end{array}$ \\
\hline $\begin{array}{l}\text { Lower arm-back } \\
\quad<30 \\
30-33 \\
34-37 \\
38-41\end{array}$ & $\begin{array}{r}34 \cdot 3 \\
14 \cdot 9 \\
6 \cdot 1 \\
5 \cdot 1\end{array}$ & $\begin{array}{r}15 \cdot 7 \\
4 \cdot 9 \\
0 \cdot 7 \\
2 \cdot 3\end{array}$ & $\begin{array}{l}27 \\
34 \\
18 \\
54\end{array}$ & $\begin{array}{r}15.4 \\
9.1 \\
4.8 \\
-\end{array}$ & $\begin{array}{l}6.6 \\
3.0 \\
1.8 \\
-\end{array}$ & $\begin{array}{r}14 \\
17 \\
8 \\
-\end{array}$ & $\begin{array}{c}11 \cdot 6 \\
8 \cdot 1 \\
-\end{array}$ & $\begin{array}{l}4 \cdot 7 \\
2 \cdot 6 \\
- \\
-\end{array}$ & $\begin{array}{l}5 \\
9 \\
- \\
-\end{array}$ & $\begin{array}{l}7 \cdot 7 \\
4 \cdot 2 \\
-\end{array}$ & $\begin{array}{l}\overline{1 \cdot 3} \\
-\end{array}$ & $\begin{array}{l}3 \\
6 \\
- \\
-\end{array}$ \\
\hline $\begin{array}{l}\text { Dorsum of hand } \\
<30 \\
30-33 \\
34-37 \\
38-41\end{array}$ & $\begin{array}{r}29.4 \\
15 \cdot 2 \\
6 \cdot 1 \\
5 \cdot 2\end{array}$ & $\begin{array}{r}10 \cdot 1 \\
4 \cdot 8 \\
1.9 \\
2.4\end{array}$ & $\begin{array}{l}26 \\
34 \\
18 \\
53\end{array}$ & $\begin{array}{r}19.0 \\
10.4 \\
5.3 \\
-\end{array}$ & $\begin{array}{l}7 \cdot 1 \\
3 \cdot 9 \\
2 \cdot 1 \\
-\end{array}$ & $\begin{array}{r}14 \\
17 \\
8 \\
-\end{array}$ & $\begin{array}{l}12 \cdot 6 \\
10 \cdot 2 \\
- \\
-\end{array}$ & $\begin{array}{l}5 \cdot 1 \\
3 \cdot 1 \\
- \\
-\end{array}$ & $\begin{array}{l}5 \\
9 \\
-\end{array}$ & $\begin{array}{r}10.3 \\
6.8 \\
-\end{array}$ & $\begin{array}{l}\overline{1.9} \\
- \\
-\end{array}$ & $\begin{array}{l}3 \\
6 \\
- \\
\end{array}$ \\
\hline
\end{tabular}


Table 2-continued

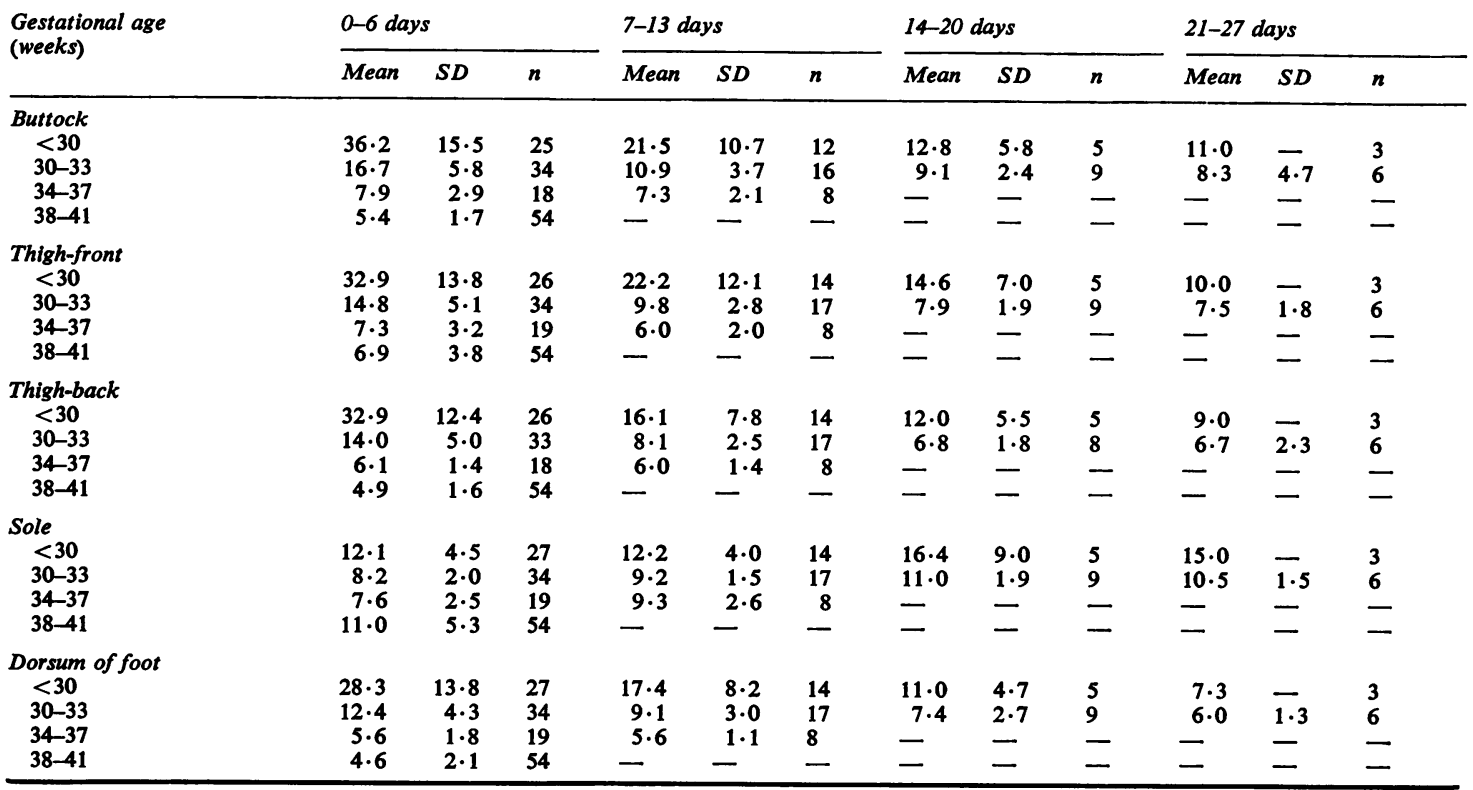

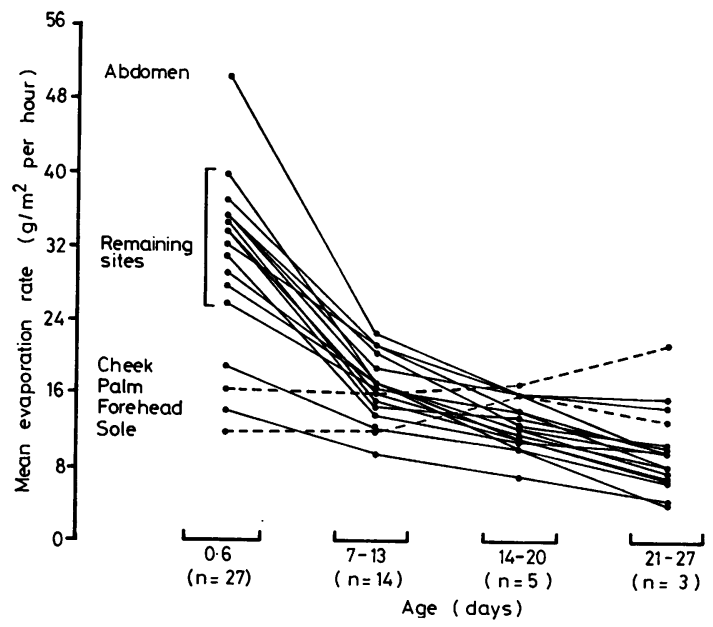

Fig. 5 Mean rates of evaporation of water at 18 skin sites for babies <30 weeks' gestation.

\section{Discussion}

It was perhaps surprising to find that the skin water loss of mature infants after the first $\mathbf{4}$ hours of life was lower than that of adults. Lamke et al. (1977) using Nilsson's method found average values in supposedly nonsweating adults to be about 12 $\mathrm{g} / \mathrm{m}^{2}$ per hour, compared with our values of $6 \mathrm{~g} / \mathrm{m}^{2}$ per hour in mature infants. In adults, over most areas water loss was about $8 \mathrm{~g} / \mathrm{m}^{2}$ per hour, but higher values of 20,35 , and $80 \mathrm{~g} / \mathrm{m}^{2}$ per hour were obtained from the forehead, soles, and palms respectively. Cunico et al. (1977) suggested that the higher values at these sites in a comfortable thermal environment were due to mild sweating. In both adults and babies, skin water loss and basal metabolic rate are directly related (Levine and Marples, 1930; Hey and Katz, 1969), so the higher water loss in adults might simply be due to the fact that they have higher metabolic rates per unit surface area than babies.

In both adults and term infants skin water loss was relatively higher from the palms of the hands and the soles of the feet (Hammarlund et al., 1977; Lamke et al., 1977) and we have confirmed this in term infants. Water loss from these sites is thought to be due mainly to sweating since the skin here is too thick to allow a high transepidermal water loss. This mental or emotional sweating is outside thermal control and is present over a wide range of ambient temperatures.

In the first 4 hours of life, the skin water loss in babies in groups 1 and 2 (34 to 41 weeks' gestation) was high and showed wide individual variation. All these babies were dried after birth with a warm towel and were not obviously wet when measured at least 2 hours later. The rates of water loss subsequently fell, suggesting that the surface of the skin was still drying out. It is well recognised that evaporative 
water loss plays a major part in the fall in body temperature which commonly occurs after birth.

Strikingly high losses were found in the immature babies. The more immature the baby, the higher was the skin water loss. Losses in babies who were light-for-dates were appropriate for their gestations. The highest skin water loss, about $60 \mathrm{~g} / \mathrm{m}^{2}$ per hour, was from a baby of 26 weeks' gestation weighing $660 \mathrm{~g}$ on the first 2 days of life. Several investigators have studied water loss in preterm infants, and various methods have been used. Hey and Katz (1969) measured the total evaporative water loss of naked term and preterm babies in a metabolic chamber and derived a skin loss from this by subtracting the measured respiratory loss. Although their study included a baby weighing $1.1 \mathrm{~kg}$ at 28 weeks' gestation, no difference in water loss between term and preterm infants was shown. However, Fanaroff et al. (1972), Wu and Hodgman (1974), and Marks et al. (1977) measured the insensible weight loss of preterm infants nursed naked in incubators using a sensitive balance and found relatively high weight losses, the average value of about $30 \mathrm{~g} / \mathrm{m}^{2}$ per hour in babies weighing $<1.25 \mathrm{~kg}$. Ambient temperature and humidity conditions were similar to ours. In magnitude these values are close to our own, which demonstrates that the higher weight losses of preterm babies are largely a consequence of water loss through the skin as opposed to the respiratory tract.

Although theoretically possible, it seems unlikely that the high losses in immature babies are a result of active sweating. Sweating in response to thermal stimuli has not been demonstrated in infants $<210$ days' postconceptual age (Foster et al., 1969). Furthermore, in our study the most immature babies in the first week of life who showed the highest water losses, had lower skin and rectal temperatures than term babies (Table 1). Term babies had low water losses and were clearly not sweating. Water loss from the skin in immature babies is therefore predominantly transepidermal. Transepidermal water loss depends on two factors: the permeability of the epidermis to water, and the environmental conditions. The ambient temperature and water vapour pressure were similar in the term and preterm babies and cannot account for the high losses. The skin and rectal temperatures were often lower in the most immature babies which would tend to reduce transepidermal water loss. The high water losses can therefore only be explained by the properties of the epidermis itself. It has been demonstrated that the water-retaining properties of the epidermis are fairly evenly distributed throughout the stratum corneum. The dried, compact cells of the stratum corneum have a much greater resistance to water diffusion than the deeper aqueous epidermal cells (Scheuplein, 1972). The fibrous protein, keratin, which is the major constituent of the stratum corneum is thought to be the main barrier to water diffusion. The skin of the preterm baby looks and feels thin, these features being used in the assessment of gestational age. In the very immature baby $<30$ weeks' gestation it appears moist and the dermal blood vessels are easily visible. The stratum corneum is poorly developed and keratinisation, even of the palms and soles, does not occur until the 6th or 7th month of fetal life (Ernst, 1896). It seems likely therefore that the high water loss of preterm babies is the result of a lack of keratin. A preterm infant is a water-dwelling fetus forced to live in air; his skin must make premature adjustments to the new air interface. The more immature the infant, the greater the leak of water, and the longer it takes after birth for the leak to stop. However, even in the most immature babies, skin water loss falls to levels similar to those found in term babies after 2 or 3 weeks. This suggests that postnatal existence hastens functional maturation of the skin, possibly by stimulating the formation of keratin. It is consistent with the observation that the skin of extremely immature babies looks similar to that of a term infant by the time the baby is about one-month old

It is interesting that the highest rate of water loss in the most immature babies was found over the abdomen where the skin looks thinner and more transparent than at any other site. Similarly, the skin of the forehead, cheek, palms, and soles, which looks thicker than elsewhere, showed a lower permeability to water. Water loss from the palms and soles increased as the babies grew older, whereas losses at all other sites decreased. Furthermore, losses from palms and soles were higher in term babies. It seems likely that a proportion of the water evaporating from the skin of the palm and the sole in preterm babies in the first month is derived from sweat glands and represents the onset of emotional sweating.

\section{Implications}

From a knowledge of the surface area and the resting oxygen consumption of a baby, it is possible to estimate the total water loss from a baby's skin and the proportion of the baby's resting heat production that this evaporative heat loss represents (Table 3). Thus a term, $3.5 \mathrm{~kg}$ baby, one-day old, nursed naked in an incubator loses about $30 \mathrm{ml}$ water from the skin each day $(8 \mathrm{ml} / \mathrm{kg}$ per day). In terms of heat loss, this is equivalent to about $10 \%$ of the resting heat production. Clearly, water loss from the skin of a term baby is not of major 
Table 3. Estimated effects of skin water loss in two babies, one term (Case 1) and one extremely immature (Case 2), aged one day. Water and evaporative heat loss are low in Case 1. Case 2 however, has a high daily water loss and his evaporative heat loss from the skin exceeds his resting heat production. Even if his heat loss by radiation and convection is abolished, he must increase his heat production in order to maintain his body temperature

\begin{tabular}{|c|c|c|}
\hline & $\begin{array}{l}\text { Case } 1 \\
(3 \cdot 5 \mathrm{~kg}, 40 \text { weeks' } \\
\text { gestation) }\end{array}$ & $\begin{array}{l}\text { Case } 2 \\
(0.9 \mathrm{~kg}, 28 \text { weeks } \\
\text { gestation })\end{array}$ \\
\hline $\begin{array}{l}\text { Surface area }\left(\mathrm{m}^{2}\right) \\
\text { (Boyd, 1935) }\end{array}$ & 0.2 & 0.1 \\
\hline $\begin{array}{l}\text { Resting oxygen consump- } \\
\text { tion }\left(\mathrm{ml} \mathrm{O} \mathrm{O}_{2} / \mathrm{kg} \mathrm{per}\right. \\
\min )(\mathrm{Hey}, 1969)\end{array}$ & $6 \cdot 5$ & 5.5 \\
\hline $\begin{array}{l}\text { Skin water loss } \\
\left(\mathrm{g} / \mathrm{m}^{2} \text { per hour }\right)\end{array}$ & 6 & 32 \\
\hline $\begin{array}{l}\text { Evaporative heat loss* } \\
\text { from the skin (watts) }\end{array}$ & 0.8 & $2 \cdot 2$ \\
\hline $\begin{array}{l}\text { Resting heat production } \dagger \\
\text { (watts) }\end{array}$ & $7 \cdot 7$ & 1.7 \\
\hline $\begin{array}{l}\text { Evaporative heat loss } \\
\text { from the skin as a } \% \\
\text { of heat production }\end{array}$ & $\frac{0.8}{7 \cdot 7} \times 100 \bumpeq 10 \%$ & $\frac{2 \cdot 2}{1 \cdot 7} \times 100=130 \%$ \\
\hline
\end{tabular}

${ }^{*}$ Latent heat of evaporation of water $580 \mathrm{cal} / \mathrm{ml}$.

$\dagger 1 \mathrm{ml} \mathrm{O} \mathrm{O}_{2}=4.83 \mathrm{cal}$.

importance once the skin has been dried after delivery. This however is not so for the very low birthweight, immature baby with a relatively high surface area in relation to body weight. A baby $<30$ weeks' gestation, weighing $0.9 \mathrm{~kg}$, one-day old, nursed naked in an incubator loses about $75 \mathrm{ml}$ water from the skin each day (about $85 \mathrm{ml} / \mathrm{kg}$ per day). In terms of evaporative heat loss, this exceeds his resting heat production. Hence, even if radiant and convective heat losses are eliminated, the baby can only maintain his body temperature by increasing his heat production. If the capacity to do this is limited, his body temperature will fall. This explains a clinical paradox. A very small immature baby nursed naked in an incubator frequently has a low rectal temperature. If radiant heat losses are eliminated by use of a Perspex radiant heat shield (Hey and Mount, 1966) and convective heat losses are abolished by raising the incubator air temperature to that of the baby's skin, the baby may still remain cold. This is because his high evaporative heat loss is greater than his maximum rate of heat production.

Controlled trials have shown that keeping small, 1-2 $\mathrm{kg}$ babies warm increases their chances of survival (Silverman et al., 1958; Day et al., 1964) and results in an increased rate of growth (Glass et al., 1969). Immature babies, $<1.0 \mathrm{~kg}$ birthweights have a very high mortality rate, while their rate of growth in the first few weeks of life is often extremely poor.
If the high evaporative water losses from the skin of these immature babies were greatly reduced, their chances of survival and their growth rates might be considerably improved.

We thank the medical and nursing staff of the Department of Neonatal Medicine and Surgery, and the midwives of ward 3A Maternity Unit, Nottingham City Hospital for help, and the Special Trustees for Nottingham University Hospitals for financial support.

\section{References}

Babson, S. G., Behrman, R. E., and Lessel, R. (1970). Fetal growth. Live born birth weights for gestational age of white, middle class infants. Pediatrics, 45, 937-944.

Boyd, E. (1935). The Growth of the Surface Area of the Human Body. University of Minnesota Press: Minneapolis, Minnesota.

Cunico, R. L., Maibach, H. I., Khan, H., and Bloom, E. (1977). Skin barrier properties in the newborn. Biology of the Neonate, 32, 177-182.

Day, R. L., Caliguiri, L., Kamenski, C., and Ehrlich, F. (1964). Body temperature and survival of premature infants. Pediatrics, 34, 171-181.

Ernst, P. (1896). Studies über normale Verhornung mit Hülfe der Grams' chen Methode. Archiv für mikroscopische Anatomie und Entwicklungsmechanik, 47, 669706.

Fanaroff, A. A., Wald, M., Gruber, H. S., and Klaus, M. H. (1972). Insensible water loss in low birth weight infants. Pediatrics, 50, 236-245.

Farr, V., Kerridge, D. F., and Mitchell, R. G. (1966). The value of some external characteristics in the assessment of gestational age at birth. Developmental Medicine and Child Neurology, 8, 657-660.

Foster, K. G., Hey, E. N., and Katz, G. (1969). The response of the sweat glands of the newborn baby to thermal stimuli and to intradermal acetylcholine. Journal of Physiology, 203, 13-29.

Glass, L., Silverman, W. A., and Sinclair, J. C. (1969). Relationship of thermal environment and caloric intake to growth and resting metabolism in the late neonatal period. Biology of the Neonate, 14, 324-340.

Hammarlund, K., Nilsson, G. E., Oberg, P. A., and Sedin, G. (1977). Transepidermal water loss in newborn infants. Acta paediatrica Scandinavica, 66, 553-562.

Hey, E. N. (1969). The relation between environmental temperature and oxygen consumption in the new-born baby. Journal of Physiology, 200, 589-603.

Hey, E. N. (1971). The care of babies in incubators. In Recent Advances in Paediatrics, fourth edition, pp. 171-216. Edited by D. Gairdner and D. Hull. Churchill: London.

Hey, E. N., and Mount, L. E. (1966). Temperature control in incubators. Lancet, 2, 202-203.

Hey, E., and Katz, G. (1969). Evaporative water loss in the newborn baby. Journal of Physiology, 200, 605-619.

Lamke, L. O., Nilsson, G. E., and Reithner, H. L. (1977). Insensible perspiration from the skin under standardized environmental conditions. Scandinavian Journal of Clinical and Laboratory Investigation, 37, 325-331.

Levine, S. Z., Kelly, M., and Wilson, J. (1930). The insensible perspiration in infancy and in childhood, II. Proposed basal standards for infants. American Journal of Diseases of Children, 39, 917-929. 
Levine, S. Z., and Marples, E. (1930). The insensible perspiration in infancy and childhood. III. Basal metabolism and basal insensible perspiration of the normal infant: a statistical study of reliability and correlation. American Journal of Diseases of Children, 40, 269-284.

Lund, C. C., and Browder, N. C. (1944). The estimation of areas of burns. Surgery, Gynecology, and Obstetrics, 79, 352-358.

Marks, K. H., Friedman, Z., and Maisels, M. J. (1977). A simple device for reducing insensible water loss in lowbirth-weight infants. Pediatrics, 60, 223-226.

Nilsson, G. E. (1977). Measurement of water exchange through skin. Medical and Biological Engineering and Computing, 15, 209-218.

Scheuplein, R. J. (1972). Properties of the skin as a membrane. In Pharmacology and the Skin. Edited by W. Montagna, E. J. Van Scott, and R. B. Stoughton. Appleton-CenturyCrofts: New York.

Silverman, W. A., Fertig, J. W., and Berger, A. P. (1958). The influence of the thermal environment upon the survival of newly-born premature infants. Pediatrics, 22, 876-886.

Thomson, A. M.. Billewicz, W. Z., and Hytten, F. E. (1968). The assessment of fetal growth. Journal of Obstetrics and Gynaecology of the Critish Commonwealth, 75, 903-916.

Wu, P. Y. K., and HoJ Jman, J. E. (1974). Insensible water loss in pre-term infants: changes with postnatal development and non-ionizin's radiant energy. Pediatrics, 54, 704-712.

Zweymuller, E., and Preinir:. O. (1970). The insensible water loss of the newborn infant. Asta psediatrica Scandinavica, Supplement 205, 1-29.

Correspondence to Dr N. Ruter Department of Child Health, Medical Schoo, Cuacn's Medical Centre, Nottingham NG7 2UH.

Received 2 August 1978 